\title{
Non-invasive Sampling for Assessment of Oxidative Stress and Pro-inflammatory Cytokine Levels in Beta- Thalassaemia Major Patients
}

\author{
Mohd. Rashdan Abd. Rahim, Jin Ai Mary Anne Tan, Ravishankar Ram Mani, \\ Umah Rani Kuppusamy*
}

\author{
Department of Biomedical Science, Faculty of Medicine, University of Malaya, 50603 Kuala \\ Lumpur, Malaysia;
}

\begin{abstract}
Background: Beta-thalassaemia ( $\beta$-thalassaemia) major patients are severely anaemic and require life-long blood transfusions for survival. These patients require iron-chelation therapy as a result of iron overload due to the monthly blood transfusions. The iron over load can cause oxidative damage and pro-inflammation and therefore, hasten mortality. Thus, regular monitoring of the oxidative stress and pro-inflammation status may be useful in these patients.

Methods: Measurement of biomarkers is usually performed on serum samples but the evaluation in non-invasive samples such as saliva would be more favourable in paediatric cases. In this study, the levels of pro-inflammatory cytokines such as tumour necrosis factor- $\alpha(T N F-\alpha)$ and interleukin-6 (IL-6) as well as oxidative indices such as lipid hydro peroxide, advanced oxidation protein products (AOPP), ferric reducing antioxidant power (FRAP), uric acid (UA) and glutathione peroxidase (GPx) activity in a total of $65 \beta$-thalassaemia major patients (all on iron chelation) and 55 healthy control subjects were assessed. All the above biochemical parameters, measured using well established assay techniques, were detectable in saliva samples.

Results: Non parametric analyses showed that lipid hydroperoxide (LH) and glutathione peroxidase (GPX) activities were significantly higher in $\beta$-thalassaemia major patients. All other parameters were not significantly different between patient and control groups implying that iron chelation therapy was successful in attenuating oxidative stress. Strong positive correlation was observed between FRAP and UA levels. There was also a notable difference in tumour necrosis factor- $\alpha(T N F-\alpha)$ between the patients and healthy controls when analysed according to ethnicity and age. AOPP level in $\beta+$-thalassaemia homozygous patients were significantly higher than $\beta+/ \beta 0$-compound heterozygous and $\beta 0$-thalassaemia homozygous patients.

Conclusion: Saliva may serve as a reliable, non-invasive sample which can be used to assess oxidative indices and pro-inflammatory cytokines in $\beta$ - thalassaemia major patients.
\end{abstract}

Keywords: $\beta$-thalassaemia major;saliva;oxidative stress; biochemical analysis

Received: 11 $1^{\text {th }}$ October 2015; Accepted: $11^{\text {th }}$ February 2016; Published: $14^{\text {th }}$ March 2016

*Corresponding author: Umah Rani Kuppusamy, Department of Biomedical Science, University of Malaya, Kuala Lumpur, Malaysia, e-mail: umah@um.edu.my 


\section{Introduction}

Beta-thalassaemia is a common genetic disorder in the Mediterranean region and South East Asian countries including Malaysia. It is estimated that $4.5 \%$ of Malaysians carry $\beta$-globin gene mutations (1). More than 3,000 patients registered with the Malaysia Thalassaemia Registry were identified with $\mathrm{HbE} / \beta$-thalassaemia and $\beta$-thalassaemia major (2). Beta thalassaemia is caused by mutations in the $\beta$-globin gene complex (3). The $\beta$-globin gene mutations cause a reduction or zero synthesis of $\beta$-globin chains. The reduction of $\beta$-globin chain production results in increase of free $\alpha$-globin chains and $\alpha$-globin chain toxicity. These will lead to erythrocyte membrane fragility and destruction of erythrocytes (4). $\beta$-thalassaemia carriers can be slightly anaemic or asymptomatic while $\beta$-thalassaemia major children are severely anaemic. Beta-thalassaemia major patients require life-long blood transfusion to maintain their haemoglobin levels higher than $9.5 \mathrm{~g} / \mathrm{dl}$ (5). However, rapid destruction of erythrocytes and hypertransfusion lead to iron overload. Even though iron chelation therapy is carried out, iron overload which leads to oxidative stress still poses a problem in thalassaemia major patients $(6,7)$. Several studies have reported the presence of oxidative stress in $\beta$-thalassaemia major patients (6-8). However, these reports used blood samples for analysis which involved invasive sampling procedures. To date, there is a lack of reports on the assessment of oxidative stress indices in $\beta$-thalassaemia major using non-invasive samples. Non-invasive sampling has gained attention due to its simplicity and convenience. Non-invasive sampling can be readily performed with minimal supervision and potential risks of adverse effects such as haematoma and nerve injury due to phlebotomy can also be avoided (9).

Non-invasive samples such as saliva have been previously used for biochemical assays (1012). A published report showed significant cor- relation between analytes present in saliva and serum samples (13). Subsequent reports have successfully compared the antioxidant indices and cytokine levels between healthy individuals and patients of various disorders using non-invasive samples (12-15). Saliva samples may serve as an alternative sample for biochemical assays especially when plasma samples cannot be obtained from subjects. It is expected that the use of non-invasive samples will be well accepted, especially by paediatric patients and parents. The success of using saliva samples in monitoring oxidative stress and pro-inflammatory status in $\beta$-thalassemia major patients may provide a good alternative platform to determine effective iron chelation therapy in these patients.

\section{Materials and Methods}

Sixty-five $\beta$-thalassaemia major patients with ages ranging from 5 to 25 -years old were recruited during their monthly blood transfusions in University Malaya Medical Centre (UMMC). These patients were categorized according to gender (25 males and 40 females), age groups [below 10-years $(\mathrm{n}=20), 11-20$-years $(\mathrm{n}=34)$ and above 20-years $(\mathrm{n}=11)]$, ethnicity [Malay $(n=37)$, Chinese $(n=20)$ and Others (consist of Indian and patients with interracial marriage in their ancestry, $n=8)$ ], and genetic classification of $\beta$-thalassaemia major ( $\beta^{+}$-thalassaemia homozygotes $\left(\beta^{+} / \beta^{+}, \mathrm{n}=9\right), \beta^{+} / \beta^{0}$-compound heterozygotes $(\mathrm{n}=39)$ and $\beta^{0}$-thalassaemia homozygotes $\left(\beta^{0} / \beta^{0}, n=17\right)(16)$. Age-matched healthy controls $(n=55)$ were also recruited from the public. Smokers and individuals with severe anaemia due to other causes, serious and chronic diseases and under drug treatment were excluded. Body mass index (BMI), defined as weight in kilograms divided by the square of height in meters was determined for each participant. BMI values of $\geq 28.0$ for men and $\geq 28.8$ for women were considered as obese as per the published criteria of the World Health Organization (WHO) 
(17). The Medical Ethics Committee (MEC) of University Malaya Medical Centre (UMMC), (MEC Ref. No: 727.1) approved this study, and conducted as per the Guidelines and Declaration of Helsinki. Written and oral consents were obtained from all patients and healthy controls or their legal guardians. Participation in this study was on a voluntary basis.

Subjects were asked to rinse their mouth with water prior to sample collection. Subsequently, the subjects expectorated at least $5 \mathrm{~mL}$ of their saliva into wide mouth $50 \mathrm{~mL}$ centrifuge tubes. The samples were subjected to centrifugation at 2,500 $\mathrm{x} g$ for 10 minutes at $4^{\circ} \mathrm{C}$. Aliquots of the resulting supernatant was stored at $-80^{\circ} \mathrm{C}$ immediately. Prior to performing the assays, the sample aliquot was thawed once and centrifuged at $10,000 \times \mathrm{g}$ for 10 minutes at $4^{\circ} \mathrm{C}$ to remove mucin. Five oxidative indices and two cytokines were measured using the saliva samples. All samples were assayed in triplicates.

The total non-enzymic antioxidant level was measured using Ferric Reducing Antioxidant Power (FRAP) assay. The measurement was based on the reduction of ferric-2,4,6-tripyridyl-triazine complex ( $\mathrm{Fe}^{3+}$-TPTZ) to the ferrous form $\left(\mathrm{Fe}^{2+}-\mathrm{TPTZ}\right)$. The standard curve obtained using ferrous sulphate heptahydrate was used to calculate the FRAP value (18).

The advanced oxidation protein product level (AOPP) was estimated by measuring the absorbance of the samples mixed with potassium iodide in acidic condition. Chloramine-T was used as standard to calculate the concentration (19).

The level of lipid peroxidation was estimated by measuring lipid hydroperoxide concentration (LH). Samples (containing malonaldehyde) were incubated at $45^{\circ} \mathrm{C}$ with methyl-phenyl indole and hydrochloric acid and the resulting coloured complex was measured spectrophotometrically at $586 \mathrm{~nm}$. The malonaldehyde present in the samples was quantitated using 1,1,3,3-tetraethoxypropane as the standard (20).
Estimation of glutathione peroxidase activity (GPx) was performed using Glutathione Peroxidase Assay Kit (Cayman Chemical Company, MI, Cat: 703102). Measurement of uric acid concentration was performed using Uric Acid Fluorometric Assay Kit (BioVision Inc, CA, Cat: K608-100). The cytokines tumour necrosis factor- $\alpha$ (TNF- $\alpha$ ) and interleukin-6 (IL-6) concentration was measured using ELISA MAX Deluxe kits (Bio Legend, CA, Cat: 43204 and 43504, respectively).

\section{Statistical analysis}

Statistical analysis was performed using SPSS version 15 (SPSS Inc., Chicago, USA). Data shown are expressed in mean \pm SEM unless indicated otherwise. Box and whisker plot was used to show distribution of salivary GPx activity and pro-inflammatory cytokine levels in $\beta$-thalassaemia patients. The significance of differences between means at a confidence limit of $95 \%$, set at $\mathrm{P}<0.05$, was assessed using one-way analysis of variance (ANOVA), followed by Tukey-Kramer test for multiple variable comparison. On the other hand, the significance of differences between median was set at $\mathrm{P}<0.05$ and assessed using Mann-Whitney $\mathrm{U}$ test. Comparison of parameters according to demographic distribution of $\beta$-thalassaemia major patients was carried out using Kruskal-Wallis H test. A Mann-Whitney U test with a significance level adjusted by a Bonferroni correction (formula $=\alpha /$ number of test) was conducted as a post-hoc test.

\section{Results}

\section{Comparison of salivary oxidative indices and cytokine levels, between $\beta$-thalassaemia major patients and healthy controls.}

In the present study, parametric analysis showed that the mean salivary LH level and GPx activity were significantly higher in the $\beta$-thalassaemia patients compared to healthy control subjects 
(Table 1). The mean salivary LH level was elevated approximately by 3 -fold $(p<0.05)$ while GPx activity was increased by $60 \%$ compared with healthy controls $(p<0.01)$. However, non parametric analysis showed that the enzyme activity was increased by $35 \%(U=781.50, p<$ 0.01). (Figure 1)

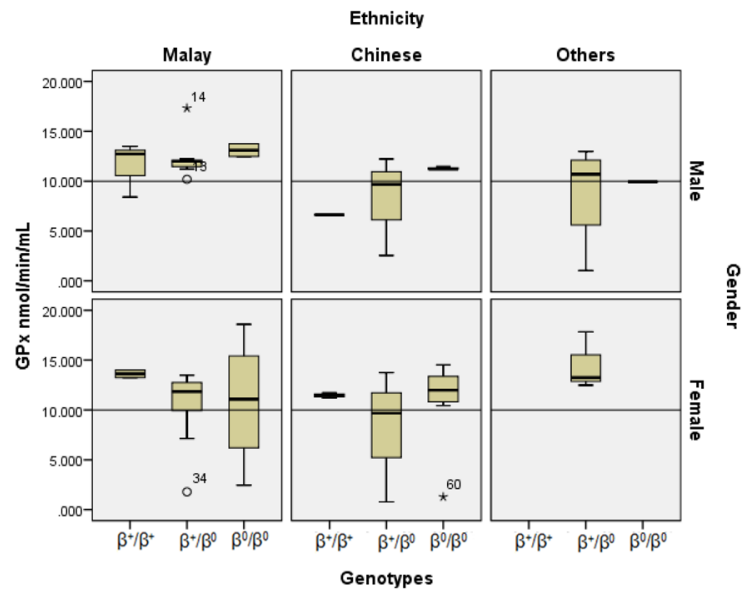

Figure 1. Whisker plot analysis of salivary GPx activity in $\beta$-thalassaemia patients compared to healthy control. Salivary GPxwas measured using Glutathione Peroxidase Assay Kit. Data represent triplicate assays for samples from patients $(n=65)$ and healthy control $(n=55)$. Level of significance was set at $\mathrm{p}<0.05$.

By assuming the data (Table 2) distribution was normal, there was a highly significant difference in salivary TNF- $\alpha$ level between the ethnic groups $(F(2,62)=6.17, p<0.01)$ and age groups $(F(2,62)=3.56, p<0.05)$. From the Tukey's post-hoc test, the level of salivary TNF- $\alpha$ was higher in patients from other ethnicity $(19.42 \pm 5.54 \mathrm{pg} / \mathrm{mL})$ compared with Malay ethnicity $(7.81 \pm 1.03 \mathrm{pg} / \mathrm{mL})$. The level of salivary TNF- $\alpha$ was also significantly higher in the patients aged between 11 - 20-years (12.86 \pm $1.92 \mathrm{pg} / \mathrm{mL}$ ) compared with patients aged above 20 -years $(4.32 \pm 1.01 \mathrm{pg} / \mathrm{mL})$. Using non-parametric statistical analysis, significant difference was observed in AOPP level depending on the genetic classification of $\beta$-thalassaemia, $(\chi 2$ (2) $=6.38, p<0.05)$. The Mann-Whitney $\mathrm{U}$ test with Bonferroni correction was conducted on this parameter with significance level set to $p$ $<0.017$ as a post-hoc test. No significance of AOPP level was observed between $\beta^{+}$-thalassaemia homozygotes patients and $\beta^{+} / \beta^{0}$-compound heterozygotes patients $(U=90.00, p=$ $0.02), \beta^{+}$-thalassaemia homozygous patients and $\beta^{0}$-thalassaemia homozygous patients $(U=$ $39.00, p<0.04)$ and between $\beta^{+} / \beta^{0}$-compound heterozygous patients and $\beta^{0}$-thalassaemia homozygous patients $(U=269.00, p<0.27)$.

TNF- $\alpha$ and IL- 6 level were significantly different between the age groups $\left(\chi^{2}(2)=7.74, p<\right.$ $0.05)$ and ethnicity $\left(\chi^{2}(2)=6.27, p<0.05\right)$. Box and whisker plot of salivary TNF- $\alpha$ \& IL- 6 distribution showed that, TNF- $\alpha$ (Figure 2A) and IL-6 (Figure 2B) levels were significantly lower in patients aged above 20-years (sub-group B) compared with patients aged below 10 -years (sub-group A) $(U=52.00, p<0.05)$ and patients aged between $11-20$-years (sub-group C) $(U$ $=87.00, p<0.01)$. The pro-inflammatory cytokine levels were also higher in $\beta$-thalassaemia major patients from Malay ethnicity compared with Chinese and other ethnicity $(U=81.50$, $p<0.05$ ).

\section{Correlation analysis between oxidative stress indices and cytokine levels in the $\beta$-thalassae- mia major patients}

Significant correlations were observed between FRAP/UA $(r=0.70, p<0.01)$, AOPP/IL-6 $(r=$ $0.31, p<0.05), \mathrm{LH} / \mathrm{UA}(r=0.26, p<0.05)$ and $\mathrm{UA} / \mathrm{TNF}-\alpha(r=0.29, p<0.05)$. Using non-parametric statistical analysis, significant correlations were observed between FRAP/UA $\left(r_{s}(63)\right.$ $=0.72, p<0.01), \mathrm{FRAP} / \mathrm{AOPP}\left(r_{s}(63)=0.43\right.$, $p<0.01)$, AOPP/UA $\left(r_{s}(63)=0.25, p<0.05\right)$, LOOH/IL-6 $\left(r_{s}(63)=-0.25, p<0.05\right)$ and UA/ $\mathrm{TNF}-\alpha\left(r_{s}(63)=-0.25, p<0.05\right)$. 

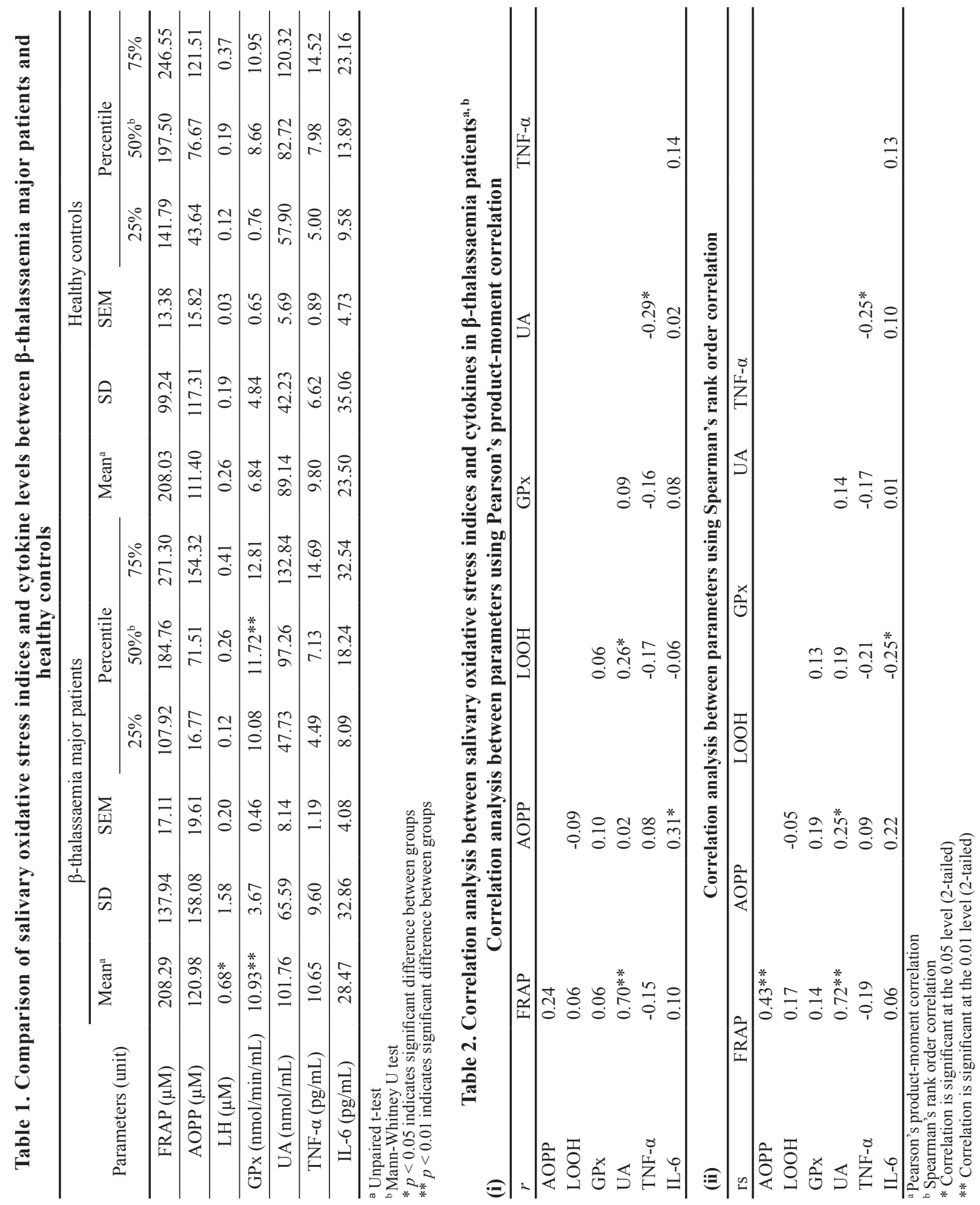
A

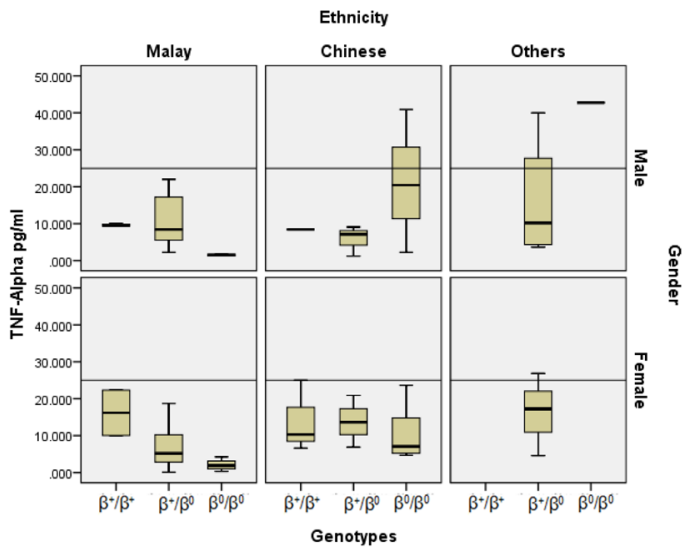

B

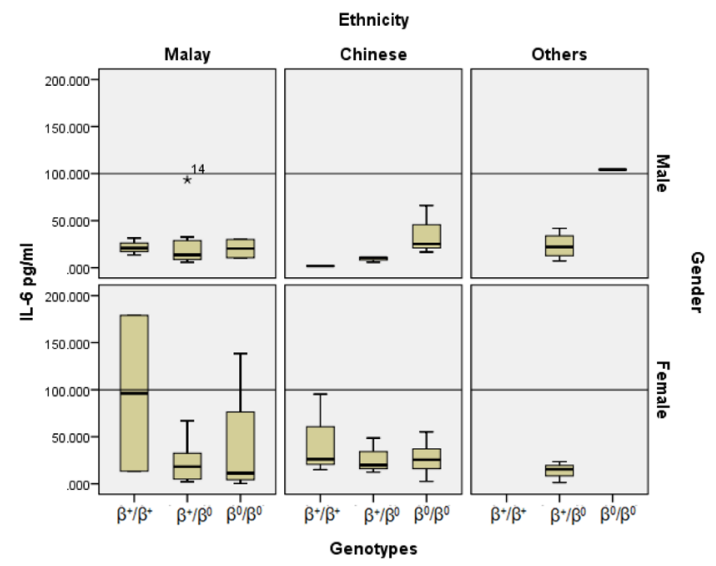

Figure 2. Whisker plot analysis of salivary proinflammatory cytokines (A) TNF- $\alpha$ and (B) IL-6 levels in $\beta$-thalassaemia patients compared to healthy control. Salivary pro-inflammatory cytokines (A) TNF- $\alpha$ (B) IL-6 levels were measured using commercial double-antibody sandwich ELISA. Data represent triplicate assays for samples from patients $(n=65)$ and healthy control $(n=55)$. Level of significance was set at $p<0.05$.Pro-inflammatory cytokines(A) TNF- $\alpha$ (B) IL-6 levels were higher in $\beta$-thalassaemia major patients from Malay ethnicity compared with Chinese and Indian ethnicity.

\section{Discussion}

The present study demonstrated that oxidative stress indices and pro-inflammatory cytokines were successfully measured using saliva samples. The enzyme GPx is one of the numerous enzymatic antioxidants preventing erythrocyte breakdown by hydroxyl radicals (21). The radicals are able to oxidize polyunsaturated lipids such as those found in the cell membrane, forming LH (22). The lipid hydroperoxides serve as substrates for selenoperoxidases which include GPx and therefore, the concurrent increase of these two parameters are expected. On the other hand, non-parametric statistical analysis showed a lack of significant difference in LH (indicator of lipid damage) and AOPP (indicator of protein damage) but an increase in GPx activity was evident when compared between patient and healthy control groups. This suggests enzymatic antioxidant was active in defending against free radical induced oxidative damage in these patients.

Although UA was usually associated with oxidative stress, it also possesses antioxidant ability $(23,24)$. This could be the factor affecting the relationship between UA and AOPP, LH and TNF- $\alpha$.

Increase in AOPP level was reported to be associated with monocyte activation (25). The increase in monocyte activation may have led to cellular damage and increased amount of purine and UA. UA, in turn may also have help in attenuating protein oxidation by preventing protein nitration as reported by an earlier publication (26). This could be the possible explanation of positive moderate correlation between UA/ AOPP using non-parametric analysis. In aspect of UA/LH relationship, the increase in LH suggests the presence of cellular damage or lysis, which increases the amount of DNA and UA due to purine metabolism released in the plasma and saliva, indirectly (27). As UA is also able to prevent further lipid peroxidation, this may help in attenuating LH production (28).

Recent experimental evidence suggests that excess plasma UA is able to induce inflammatory cytokines including TNF- $\alpha$ in medical con- 
ditions (29). The salivary UA provides minimal contribution in reducing salivary TNF- $\alpha$ production. The attenuation of protein oxidation and lipid peroxidation may be attributed to UA (antioxidant) and this could prevent more cellular damage and reduce pro-inflammatory cytokine production. The non-parametric statistical analysis in the present study showed that reduction of $\mathrm{LH}$ provided a weak induction to increase the IL-6 secretion (30). IL-6 has been reported to exhibit anti-inflammatory properties as well, by preventing further destruction of epithelial cells (31). This is possibly due to the IL-6 ability to induce cells to enter mitosis (32) and therefore, one should not rule out the possibility that the decreased LOOH level could favour IL-6 to induce cell proliferation.

The level of oxidative indices may differ depending on the ethnicity as observed in previous investigations $(33,34)$. Some minor differences in the food intake preferences may exist among the different ethnic groups in Malaysia and this may indirectly affect the level of antioxidants especially in the current patient cohort.

In the present study, the TNF- $\alpha$ level compared between ethnicities showed that the low sample size ( 8 of 'other' vs 37 of Malays) might have played a major role in the observation. Both parametric and non-parametric statistical analyses are sensitive to a huge difference in sample size. The duration of treatment and compliance towards chelation therapy could be the factor affecting the cytokine level between age groups. Poor compliance towards chelation treatment will not remove the accumulated iron adequately and thus, cause complications in patients (35). Due to inadequate removal of excess iron, redox imbalance still occurs and patients may still be under oxidative stress.

External factors which are not assessed such as co-inheritance of $\alpha$-globin gene mutations and polymorphisms such as $\mathrm{XmnI}$ which are able to reduce the amount of free $\alpha$-globin chains may have helped in reducing the oxidative stress severity (36). Salivary fluid component comprised of gingival crevicular fluid also contains serum exudates. This fluid was further mixed with other fluids from salivary glands and bronchial and nasal secretions (37) which could result in analyte dilution. Therefore, it is possible that the concentration or activity of the salivary oxidative indices and cytokine were lower compared with findings reported with plasma levels (38).

A pervious study on $\beta$-thalassaemia major patients attending monthly blood transfusion in UMMC showed that iron overload and oxidative stress were still observed in the patients although chelation therapy was prescribed (6). However, the patients recruited in the previous study were on single iron chelation therapy using deferoxamine (DFO). In the present study, 23\% of the patients had undergone combination iron chelation treatment involving combination of DFO and deferiprone (DFP), DFO and deferasirox (DFX) or DFP and DFX.

Published data has reported better compliance with combination chelation therapy (39) and the patients also showed improvement in endocrine glands' and cardiac functions. In addition, clinical observation also reported that DFX alone may reduce oxidative stress in transfusion dependent patients (40). These factors could be the contributors to the improvement of the oxidative stress status in $\beta$-thalassaemia major patients. Compared with diets of the healthy controls, $48 \%$ of the $\beta$-thalassaemia major patients were prescribed with supplementary vitamins such as Vitamins C, D, B12 and folic acid. Vitamins such as ascorbic acid may contribute to the estimation of total non-enzymic antioxidant status/level by FRAP assay $(41,42)$. The supplement may have helped increase the antioxidant ability in $\beta$-thalassaemia major patients to the level almost similar to healthy controls. 


\section{Conclusion}

In this study, the measurement of oxidative stress indices and cytokine levels of $\beta$-thalassaemia major patients and healthy controls could be successfully carried out using non-invasive saliva samples. The analytes of interest were within detectable range and could be measured using in-house methods and commercial assay kits. The oxidative indices among patients were mostly comparable to healthy controls probably due to better compliance towards chelation therapy, combination chelation therapy and good patient management.

\section{Disclosures: None}

\section{Acknowledgments and financial support}

This project was funded by University of Malaya Postgraduate Research Fund (PPP) PS1992009C.

\section{Authors Contribution}

MRAR performed the biochemical assays and prepared the draft of the article. URK and JAMAT designed this study and revised the article draft for correction. URK and MRSR revised the statistical analysis for this article. All authors have read and approved the final version of this manuscript.

\section{Conflict of Interest}

The authors declare that they have no competing interests.

\section{Abbreviations \\ (AOPP) advanced oxidation protein product; ( $\beta$-thalassaemia) beta-thalassaemia; $(\beta$-globin) beta - globin; (BMI) Body mass index; (DFO) deferoxamine; (DFX) deferasirox; (FRAP) Fer-}

ric Reducing Antioxidant Power; (GPx) glutathione peroxidase; (IL) Interleukins; (LH) lipid hydro peroxide; (TNF- $\alpha$ ) tumor necrosis factoralpha; (UA) uric acid; (UMMC - HEC) University of Malaya Medical Centre - Human Ethical Committee; (WHO) World Health Organisation.

\section{References}

1. George E, Tan JAMA. Genotype-phenotype diversity of beta-thalassemia in Malaysia: treatment options and emerging therapies. Med J Malaysia. 2010; 65(4):25660.

2. Abdul WJ, Naznin M, Nora MZ, Suzanah AR, Zulaiha M, Aidil Faszrul AR, Kamaruzaman WS. Thalassaemia: a study on the perception of patients and family members. Med J Malaysia. 2011;6(4):326-34.

3. Bank A. Understanding globin regulation in $\beta$-thalassemia: it's as simple as $\alpha, \beta, \gamma, \delta$. J Clin Invest. 2005 Jun 1; 115(6): 1470-3. DOI: 10.1172/JCI25398

4. Liu S, McConnell SC, Ryan TM. Erythropoiesis in the Absence of Adult Hemoglobin. Mol Cell Biol. 2013 Jun;33(11):2241-51. DOI: 10.1128/MCB.01734-12

5. Cappellini MD, Cohen A, Eleftheriou A, et al. Guidelines for the Clinical Management of Thalassaemia [Internet]. 2nd Revised edition. Nicosia (CY): Thalassaemia International Federation; 2008. Chapter 2, Blood Transfusion Therapy in $\beta$-Thalassaemia Major.

6. Kuppusamy UR, Tan JAMA. Chelation therapy with Desferrioxamine does not normalize ferritin level but attenuates oxidative damage and improves total antioxidant level in Malaysian Chinese $\beta$-thalassaemia major patients. West Indian Med J. 2011;60(1):3-8.

7. Walter PB, Macklin EA, Porter J, Evans P, Kwiatkowski JL, Neufeld EJ, Coates T, Gardina PJ, Vichinsky E, Olivieri N, Alberti D, Holland J, Harmatz, P. Inflammation and oxidant-stress in $\beta$-thalassemia patients treated with iron chelators deferasirox (ICL670) or deferoxamine: an ancillary study of the Novartis CICL670A0107 trial. Haematologica. 2008;93(6):817-25. DOI: $10.3324 /$ haematol.11755

8. Metwalley KA, El-Saied ARAH. Glucose homeostasis in Egyptian children and adolescents with $\beta$-thalassemia major: relationship to oxidative stress. Indian J Endocrinol Metab. 2014;8(3):333-9. DOI: 10.4103/22308210.131169

9. Rayegani SM, Azadi A. Lateral antebrachial cutaneous nerve injury induced by phlebotomy. J Brachial Plex Peripher Nerve Inj. 2007; 2:6-8.

10. Hegde MN, Hegde ND, Ashok A, Shetty S. Evaluation of total antioxidant capacity of saliva and serum in caries-free and caries-active adults: an in-vivo study. In- 
dian J Dent Res. 2013;24:164-7. DOI: 10.4103/09709290.116670

11. Shetty SR, Babu S, Kumari S, Shetty P, Hegde S, Castelino R. Status of salivary lipid peroxidation in oral cancer and precancer. Indian J Med Paediatr Oncol. 2014;35(2):156-8. DOI: 10.4103/0971-5851.138990

12. Soukup M, Biesiada I, Henderson A, Idowu B, Rodeback D, Ridpath $\mathrm{L}$, et al. Salivary uric acid as a non-invasive biomarker of metabolic syndrome. Diabetol Metab Syndr. 2012;4(1):14. DOI: 10.1186/17585996-4-14

13. Sculley DV, Evans SCL. Salivary antioxidants and periodontal disease status. Proc Nutr Soc. 2002;61:137-43. DOI: 10.1079/PNS2001141

14. Suh KI, Kim YK, Kho SH. Salivary levels of IL-1 $\beta$, IL-6, IL-8 and TNF- $\alpha$ in patients with burning mouth syndrome. Arch Oral Biol. 2009;54:797-802. DOI: 10.1016/j.archoralbio.2009.05.007

15. Wolfram R, Oguogho A, Palumbo B, Sinzinger H. Enhanced oxidative stress in coronary heart disease and chronic heart failure as indicated by an increased 8-epi-PGF2 $\alpha$. Eur J Heart Fail. 2005;7:167-72. DOI: 10.1016/j.ejheart.2004.05.007

16. Olivieri NF, Pakbaz Z, Vichinsky E. Hb E/beta-thalassaemia: a common \& clinically diverse disorder. The Indian Journal of Medical Research. 2011;134(4):52231.

17. Devaux M. In: Exploring the Relationship between Education and Obesity, OECD. Economic Studies. 2011;1.

18. Sudan R, Bhagat M, Gupta S, Singh J, Koul A. Iron (FeII) Chelation, Ferric Reducing Antioxidant Power, and Immune Modulating Potential of Arisaema jacquemontii (Himalayan Cobra Lily). BioMed Res Int. 2014;2014, Article ID 179865, 7 pages.

19. Marsche G, Frank S, Hrzenjak A, Holzer M, Dirnberger S, Wadsack C, et al. Plasma-Advanced Oxidation Protein Products Are Potent High-Density Lipoprotein Receptor Antagonists In Vivo. Circ Res. 2009 Mar 27;104(6):750-7. DOI: 10.1161/CIRCRESAHA. 108.193169

20. Siddique YH, Ara G, Afzal M. Estimation of Lipid Peroxidation Induced by Hydrogen Peroxide in Cultured Human Lymphocytes. Dose-Response. 2012; 10(1):1-10. DOI: 10.2203/dose-response.10-002.Siddique

21. Rahman K. Studies on free radicals, antioxidants, and co-factors. Clinical Interventions in Aging. 2007:2(2):219-36.

22. POWERS SK, JACKSON MJ. Exercise-Induced Oxidative Stress: Cellular Mechanisms and Impact on Muscle Force Production. Physiol Rev. 2008 Oct; 88(4): 1243-76. DOI: 10.1152/physrev.00031.2007

23. Duplancic D, Kukoc-Modun L, Modun D, Radic N.
Simple and Rapid Method for the Determination of Uric Acid-Independent Antioxidant Capacity. Molecules. 2011 Aug 17;16(8):7058-68. DOI: 10.3390/molecules 16087058

24. Fabbrini E, Serafini M, Colic Baric I, Hazen SL, Klein S. Effect of Plasma Uric Acid on Antioxidant Capacity, Oxidative Stress, and Insulin Sensitivity in Obese Subjects. Diabetes. 2014;63(3):976-81. DOI: $10.2337 / \mathrm{db} 13-1396$

25. Taylor EL, Armstrong KR, Perrett D, Hattersley AT, Winyard PG. Optimisation of an Advanced Oxidation Protein Products Assay: Its Application to Studies of Oxidative Stress in Diabetes Mellitus. Oxidative Medicine and Cellular Longevity. 2015;2015, Article ID 496271, 10 pages.

26. Daiber A, Daub S, Bachschmid M, Schildknecht S, Oelze M, Steven S, et al. Protein Tyrosine Nitration and Thiol Oxidation by Peroxynitrite-Strategies to Prevent These Oxidative Modifications. Int J Mol Sci. 2013 Apr 8;14(4):7542-70. DOI: 10.3390/ ijms 14047542

27. Fisher-Wellman K, Bloomer RJ. Acute exercise and oxidative stress: a 30 year history. Dyn Med. 2009 Jan 13;8:1. DOI: 10.1186/1476-5918-8-1

28. Lobo V, Patil A, Phatak A, Chandra N. Free radicals, antioxidants and functional foods: Impact on human health. Pharmacogn Rev. 2010 Jul-Dec; 4(8): 118126. DOI: $10.4103 / 0973-7847.70902$

29. Tuttolomondo A, Pecoraro R, Pinto A. Studies of selective TNF inhibitors in the treatment of brain injury from stroke and trauma: a review of the evidence to date. Drug Des Devel Ther. 2014 Nov 7;8:2221-38. DOI: $10.2147 /$ DDDT.S67655

30. Ajuwon OR, Oguntibeju OO, Marnewick JL. Amelioration of lipopolysaccharide-induced liver injury by aqueous rooibos (Aspalathus linearis) extract via inhibition of pro-inflammatory cytokines and oxidative stress. BMC Complementary and Alternative Medicine 2014;14:392. DOI: 10.1186/1472-6882-14-392

31. de Cássia da Silveira e Sá R, Andrade LN, de Sousa DP. A Review on Anti-Inflammatory Activity of Monoterpenes. Molecules. 2013;18:1227-54. DOI: $10.3390 /$ molecules 18011227

32. Puebla-Osorio N, Damiani E, Bover L, Ullrich SE. Platelet-activating factor induces cell cycle arrest and disrupts the DNA damage response in mast cells. Cell Death Dis. 2015 May 7;6:e1745.

33. Fisher G, Alvarez JA, Ellis AC, Granger WM, Ovalle $\mathrm{F}$, Man $\mathrm{CD}$, et al. Race differences in the association of oxidative stress with insulin in African- and European-American women. Obesity (Silver Spring). 2012 May;20(5):972-7. DOI: 10.1038/oby.2011.355

34. Kuppusamy UR, Indran M, Rokiah P. Glycaemic control in relation to xanthine oxidase and antioxidant 
indices in Malaysian type 2 diabetes patients. Diabet Med. 2005 Oct;22(10):1343-6. DOI: 10.1111/j.14645491.2005.01630.x

35. Flora SJS, Pachauri V. Chelation in Metal Intoxication. Int J Environ Res Public Health. 2010 Jul;7(7):274588. DOI: 10.3390/ijerph7072745

36. Chinelato, IS, Carrocini, GCdS, \& Bonini-Domingos, CR. (2011). XmnI polymorphism frequency in heterozygote beta thalassemia subjects and its relation to fetal hemoglobin levels. Rev Bras Hematol Hemoter. 2011; 33(6):483. DOI: 10.5581/1516-8484.20110128

37. Manu Rathee, Mohaneesh Bhoria. Application of saliva as diagnostic bio-fluid an overview. Journal of Diagnostics. 2014;1(2):13-7.

38. Fischer SG, Perez RS, Nouta J, Zuurmond WW, Scheffer PG. Oxidative Stress in Complex Regional Pain Syndrome (CRPS): No Systemically Elevated Levels of Malondialdehyde, F2-Isoprostanes and $8 \mathrm{OHdG}$ in a Selected Sample of Patients. Int J Mol Sci. 2013 Apr 10;14(4):7784-94 DOI: 10.3390/ijms14047784
39. Hatzipantelis ES, Karasmanis K, Perifanis V, Vlachaki E, Tziomalos K, Economou M. Combined chelation therapy with deferoxamine and deferiprone in $\beta$-thalassemia major: compliance and opinions of young thalassemic patients. Hemoglobin. 2014;38(2):111-4. DOI: 10.3109/03630269.2013.867407

40. Kochhar H, Leger CS, Leitch HA. Durable Red Blood Cell Transfusion Independence in a Patient with an MDS/MPN Overlap Syndrome Following Discontinuation of Iron Chelation Therapy. Case Reports in Hematology. 2015;2015: Article ID 253294, 7 pages.

41. Shalaby EA, Shanab SMM. Antioxidant compounds, assays of determination and mode of action. African Journal of Pharmacy and Pharmacology. 2013;7(10):52839. DOI: $10.5897 / A J P P 2013.3474$

42. Mohamed R, Campbell J-L, Cooper-White J, Dimeski G, Punyadeera C. The impact of saliva collection and processing methods on CRP, IgE, and Myoglobin immunoassays. Clinical and Translational Medicine. 2012;1:19. DOI: 10.1186/2001-1326-1-19 